\title{
Repetition counts: repeated exposure increases intake of a novel vegetable in UK pre-school children compared to flavour-flavour and flavour-nutrient learning
}

\author{
Samantha J. Caton ${ }^{1}$, Sara M. Ahern ${ }^{1}$, Eloise Remy ${ }^{2}$, Sophie Nicklaus ${ }^{2}$, Pam Blundell ${ }^{1}$ and \\ Marion M. Hetherington ${ }^{1 *}$ \\ ${ }^{1}$ Institute of Psychological Sciences, University of Leeds, Leeds LS2 9JT, UK \\ ${ }^{2}$ INRA, UMR1324, Centre des Sciences du Goût et de l'Alimentation, F-21000 Dijon, France
}

(Submitted 29 March 2012 - Final revision received 26 July 2012 - Accepted 26 July 2012 - First published online 30 October 2012)

\section{Abstract}

Children are not consuming sufficient amounts of fruits and vegetables in their habitual diet. Methods derived from associative learning theories could be effective at promoting vegetable intake in pre-school children. The objective of the present study was to compare the effectiveness of different learning strategies in promoting the intake of a novel vegetable. Children aged between 9 and 38 months were recruited from UK nurseries. The children $(n 72)$ were randomly assigned to one of three conditions (repeated exposure, flavourflavour learning or flavour-nutrient learning). Each child was offered ten exposures to their respective version of a novel vegetable (artichoke). Pre- and post-intervention measures of artichoke purée and carrot purée (control vegetable) intake were taken. At pre-intervention, carrot intake was significantly higher than artichoke intake $(P<0.05)$. Intake of both vegetables increased over time $(P<0.001)$; however, when changes in intake were investigated, artichoke intake increased significantly more than carrot intake $(P<0 \cdot 001)$. Artichoke intake increased to the same extent in all three conditions, and this effect was persistent up to 5 weeks post-intervention. Five exposures were sufficient to increase intake compared to the first exposure $(P<0 \cdot 001)$. Repeated exposure to three variants of a novel vegetable was sufficient to increase intake of this vegetable, regardless of the addition of a familiar taste or energy. Repetition is therefore a critical factor for promoting novel vegetable intake in pre-school children.

\section{Key words: Repeated exposure: Vegetable intake: Learning mechanisms: Pre-school children}

Children in the UK are not consuming sufficient amounts of fruits and vegetables in order to obtain health-related benefits $^{(1,2)}$. Many methods have been implemented to promote the intake of vegetables in children including modelling by significant others ${ }^{(3-5)}$, vegetables offered as a first course $^{(6)}$, larger portion sizes ${ }^{(7)}$ or with an accompanying $\operatorname{dip}^{(8,9)}$ and offering vegetables by stealth ${ }^{(10,11)}$.

Conditioning is an important mechanism in the development of food preferences ${ }^{(12)}$. Young children consistently show a preference for foods that are higher in energy density, in particular, foods that are high in fat ${ }^{(13-15)}$. These studies indicate that flavour-nutrient learning (FNL) might be an effective strategy for increasing intake of vegetables. FNL investigations generally involve pairing a novel flavour or food with a high-energy, flavourless ingredient such as maltodextrin or oil. Zeinstra et al. $^{(16)}$ attempted to increase vegetable liking using FNL. The experiment was unsuccessful because the children consumed insufficient amounts of the vegetable juice, despite the added energy; they disliked the taste of the juice, rating it as very intense. Thus, adding energy without altering the taste of the vegetable is not sufficient to improve liking or increase intake ${ }^{(16)}$.

Flavour-flavour learning (FFL), in which a novel flavour is paired with an already familiar and liked flavour, may also be useful in the promotion of vegetable liking and intake. FFL has been researched in rats ${ }^{(17-19)}$ and in adult human subjects $^{(20-24)}$, but fewer studies have involved children, despite this being a key time in development to acquire preferences. Using FFL, Havermans \& Jansen ${ }^{(25)}$ reported an increase in rated preference for a moderately liked vegetable juice at post-test that was previously paired with dextrose (conditioned stimulus; CS+). However, this investigation was limited by a small sample size, reliance on preference ratings only and no indication of intake.

Mere exposure ${ }^{(26)}$ is a process by which experience of a stimulus can positively enhance the individual's attitudes towards it, resulting in the given stimulus acquiring positive valence. Alternatively, repeated exposure (RE) might lead to

Abbreviations: FFL, flavour-flavour learning; FNL, flavour-nutrient learning; RE, repeated exposure.

* Corresponding author: Professor M. M. Hetherington, email m.hetherington@leeds.ac.uk 
habituation to the neophobic response ${ }^{(27)}$. Exposure is an effective way of increasing the liking and intake of novel foods in pre-school children ${ }^{(13,28-30)}$ and older children ${ }^{(31,32)}$. $\mathrm{RE}$ is also effective outside the laboratory ${ }^{(33)}$.

Few studies have investigated the relative effectiveness of different learning strategies in promoting vegetable intake in children. Tangible rewards and social praise appear to be highly effective strategies ${ }^{(1,34)}$, but modifications of the food may be sufficient to increase liking without additional external rewards. Therefore, the aim of the present investigation was to compare the effectiveness of different learning strategies (FFL, FNL) with repeated taste exposure on increasing intake of a novel vegetable in UK pre-school children.

\section{Method}

\section{Participants}

Parents of pre-school children aged 6-36 months were invited to take part in the study. All children reported to have any food allergies were excluded from taking part in the investigation. Recruitment took place (February-May 2011) in private day care nurseries in West and South Yorkshire, UK. In the first instance, nursery managers were given details of the study to check their interest in the study. If the nursery managers expressed an interest, then the participant information sheets and consent forms were distributed to parents. A total of twenty-six nurseries were approached and ten agreed to take part. In all, six nurseries started and completed the investigation, one dropped out due to lack of recruitment of children, two due to other commitments and one nursery terminated the experiment because the children would not consume the products offered. From the six nurseries that took part, 108 children aged 9-38 months were recruited. The present study was conducted according to the guidelines laid down in the Declaration of Helsinki and all procedures involving human subjects/ patients were approved by the Institute of Psychological Sciences (University of Leeds) ethics committee (10 189-02). Written informed consent was obtained from all parents and caregivers of the participating children.

\section{Procedure}

During the pre-intervention period, participants were offered up to two pots $(200 \mathrm{~g})$ of puréed artichoke first (target vegetable: RE recipe) or two pots ( $260 \mathrm{~g}$ ) of puréed carrot (control vegetable) first, then $2-3 \mathrm{~d}$ later children were offered the alternative vegetable and this was counterbalanced. Participants were offered these either mid-morning, prior to lunch (around 11.00 hours), or as a mid-afternoon snack (14.00 hours), when it would be expected that the children would be hungry. Children were always tested at the same time of day. Participants were offered the pots of vegetable by the experimenters and the nursery staff who were familiar to the children, and they were invited to consume as much or as little as they liked; if the first pot was completely consumed then the second pot was offered. It was explained to the staff and to the children that if they did not wish to eat the vegetables offered or if they did not wish to take part in the study then this was fine. Different children have different eating rates; therefore, each child was given as long as they needed to consume the vegetables. Most children ate what they wanted within approximately $15 \mathrm{~min}$. Nursery staff were instructed to feed the children the same way that they would usually feed them and rely on their own knowledge of the child to judge when the child had reached satiation. The staff were blinded to the version of the target vegetable being offered to the children. In the case where experimenters fed the children, satiation was assumed to be reached when three consecutive refusals were displayed. Refusals were typically classified as head turning, pursed lips and general disengagement with the foods offered. Children took part in the study in groups of about two to six sitting around a table at any time which was advised by nursery managers as most natural and comfortable for the children.

Nursery staff were instructed to avoid making any negative comments regarding the food's smell, taste, texture or appearance and also to avoid any negative facial responses throughout the whole experiment ${ }^{(35)}$. For the intervention period, the children were assigned to one of three conditions, i.e. RE, FNL or FFL. Table 1 contains the nutrient and energy information for each condition. Around $2-4 \mathrm{~d}$ after the pre-intervention

Table 1. Nutritional composition and recipes of the three studied artichoke purées: repeated exposure (RE), flavour-flavour learning ( $F F L)$, flavour-nutrient learning (FNL) and of the control carrot purée

\begin{tabular}{|c|c|c|c|c|}
\hline & RE & $\mathrm{FFL}$ & FNL & Carrot \\
\hline \multicolumn{5}{|c|}{ Nutritional composition (per $100 \mathrm{kcal}$ ) } \\
\hline Protein $(\mathrm{g})$ & 1.1 & $1 \cdot 1$ & $1 \cdot 2$ & 0.3 \\
\hline Total carbohydrates $(\mathrm{g})$ & 8.9 & $11 \cdot 1$ & $10 \cdot 7$ & 4.5 \\
\hline Total lipids (g) & $1 \cdot 0$ & 0.3 & $10 \cdot 7$ & 0.4 \\
\hline $\mathrm{Na}(\mathrm{mg})$ & $182 \cdot 3$ & $135 \cdot 0$ & $136 \cdot 0$ & $40 \cdot 0$ \\
\hline \multicolumn{5}{|l|}{ Energy (kcal) } \\
\hline kcal & 48 & 51 & 144 & 27 \\
\hline $\mathrm{kJ}$ & 201 & 213 & 602 & 113 \\
\hline \multicolumn{5}{|l|}{ Recipe (g/100 g) } \\
\hline Artichoke & 78.9 & $76 \cdot 2$ & $78 \cdot 3$ & \\
\hline Water of cooking & $19 \cdot 0$ & $20 \cdot 1$ & $9 \cdot 0$ & \\
\hline Oil & $1 \cdot 0$ & 0.0 & $11 \cdot 6$ & \\
\hline Sugar & 1.0 & 3.6 & 1.0 & \\
\hline Salt & 0.1 & 0.1 & 0.1 & \\
\hline
\end{tabular}


period, each child was offered one pot $(100 \mathrm{~g})$ of artichoke for ten exposures. During the post-intervention period, children were again offered both pots of carrot and artichoke on separate occasions, 2-3d apart. Durability of effect was assessed by offering each child two pots of artichoke once per week for 3 weeks, and this was offered 2 weeks from the end of the study period. On the last week (after 5 weeks), children were also offered two pots of carrot. All pots were weighed before and after to determine intake $(\mathrm{g})$ throughout the experiment. Any spillage on tables and bibs were collected after the session and were added back in to the pots before re-weighing. Children's heights and weights were recorded at the end of the intervention.

\section{Study foods}

In order to identify a novel vegetable, a questionnaire featuring fifty-six vegetables was given to seventy-one UK caregivers of pre-school children aged 6-36 months. The questionnaire asked 'Are you familiar with this vegetable?', 'How often do you eat this vegetable?', 'Have you ever offered this vegetable to your child?', 'How often do you offer this vegetable to your child?' and 'How much does your child like this vegetable?'. As a result, artichoke was chosen as one of the least familiar and least consumed vegetables from the questionnaire, with only $17 \%$ of the sample ever having been offered artichoke. When artichoke was offered to a child, it was only reported to be offered about once per month (Ahern et al. ${ }^{(36)}$ ). In addition, pure artichoke in baby food format is not easily available in the UK, so it is likely that this food was relatively unfamiliar to the majority of children enrolled in the present study.

For the development of the study food recipes, only babyfood-grade ingredients were used in order to meet the European regulation (Directive 2006/125/CE), because the study was conducted with children younger than 3 years of age.

One recipe was developed for each condition (Table 1). The RE recipe was a basic vegetable purée. To explore the FFL mechanism, the chosen unconditioned stimulus was sweetness. The selected sweet ingredient was sucrose. To explore FNL, the chosen unconditioned stimulus was a higher energy density. The selected energy-dense ingredient was sunflower oil, because of its relatively neutral taste.

To sum up, the ingredients selected were plain baby-foodgrade frozen artichoke heart (France Recherche \& Développement (FRDP)), water, sucrose (Vermandoise), sunflower oil (Huileries de Lapalisse) and salt.

The three purée recipes were developed in the Centre des Sciences du Goût et de l'Alimentation, according to the following constraints: the RE and the FFL variants should have similar energy densities, but the FFL variant ought to have a sweeter taste; the RE and the FNL variants ought to have comparable sensory properties, but different energy densities. Informal tastings with faculty members helped to define the recipes at the laboratory. Next, the production took place in a food factory (Freshinov) accredited to prepare baby foods, using appropriate ingredients and processes following baby food industry regulations. A first test of industrial production was organised, which helped to adjust the recipes, and then the study foods were produced in the appropriate amounts from the same initial batch of fresh vegetables to limit differences by seasonal variation.

For each recipe, ingredients were steamed for $20 \mathrm{~min}$ at $90^{\circ} \mathrm{C}$, mixed, conditioned in a 100 ( $\operatorname{sem} 2$ ) g jar with lid and sterilised at $120^{\circ} \mathrm{C}$ for $75 \mathrm{~min}$ at 2 bars. Bacteriological analyses were conducted by the Departmental Laboratory of Analysis and Research (Barenton-Bugny), and the nutritional composition was determined by a certified laboratory (INZO).

Baby food carrot purée, used as the control vegetable, was supplied by the Nestlé group (NaturNes ${ }^{\circledR}$; Nestlé).

\section{Sensory profile}

A sensory description of all study samples was conducted by a trained panel, consisting of twelve adults aged between 28 and 67 years. They received more than forty sessions (1-1 h $30 \mathrm{~min}$ ) of training on taste description and on the way to use the scoring scales. The referents of the Spectrum ${ }^{\mathrm{TM}}$ intensity scales for descriptive analysis were used to rate the intensity of sweet, salty, sour and bitter tastes. A 'Spectrum-like' scale was developed for fattiness perception. The panellists were asked to score the perceived intensities of each variant monadically on a linear scale, from 'not perceived' (left anchor, converted into 0) to 'very intense' (right anchor, converted into 10) using FIZZ software (Biosystèmes). The study samples were presented according to a Latin square design and two replications were performed.

\section{Non-eaters and plate clearers}

To investigate individual differences in response to the intervention, three groups of children were identified as regular eaters, non-eaters and plate clearers. Regular eaters showed a linear increase in intake over time. Non-eaters were classified as those children who consumed less than $10 \mathrm{~g}(<10 \%)$ of the amount offered over the exposure periods. Plate clearers were classified as those children who consumed on average $90 \mathrm{~g}$ or more over the exposure period and consumed more or less the entire amount from exposures 2 or 3 onwards.

\section{Anthropometrics}

Children's heights and weights were measured at the end of the intervention in those children whose parents consented ( $n$ 45). Weights were measured using digital scales (Seca) and height measured using a portable stadiometer (Leicester SMSSE-0260; Seca). Weight-for-height $z$-scores were calculated using the WHO anthropometric calculator (http://www.who. int/childgrowth/software/en/).

\section{Statistical analysis}

Data are presented as means with their standard errors. Mixed ANOVA was carried out on intake data (absolute $g$ and change $/ \Delta$ intake) with vegetable (carrot and artichoke) and 
Table 2. Characteristics (age, weight-for-height BMI $z$-scores) of children involved in the study (Mean values with their standard errors and ranges)

\begin{tabular}{|c|c|c|c|c|c|c|c|c|}
\hline & \multicolumn{4}{|c|}{ Age (months) } & \multicolumn{4}{|c|}{ BMI z-score } \\
\hline & $n$ & Mean & SEM & Range & $n$ & Mean & SEM & Range \\
\hline Males & 32 & $25 \cdot 8$ & 1.4 & $9-35$ & 16 & $1 \cdot 2$ & .18 & $0.1-2 \cdot 3$ \\
\hline Females & 40 & $21 \cdot 7$ & 1.2 & $10-38$ & 31 & $1 \cdot 1$ & 0.1 & $-0.12-2.6$ \\
\hline Overall & 72 & $23 \cdot 6$ & 0.9 & $9-38$ & $47^{*}$ & $1 \cdot 1$ & 0.1 & $-0.1-2.6$ \\
\hline
\end{tabular}

*Only forty-seven out of a possible seventy-two had their height and weight measured.

time (pre/post and 5 weeks post-intervention) as within-subject factors and condition (RE/FNL/FFL) and age group (aged less than 24 months and 24-38 months) as between-subjects factors. To examine the impact of elevated carrot intake, compared to artichoke at baseline, baseline carrot intake was included in an ANCOVA as the covariate to further investigate changes in intake pre- and post-intervention. One-way ANOVA was carried out to investigate further significant interactions between age, time and vegetable. ANCOVA was carried out on intake data over the ten exposures, with condition as the between-subject factor and change in carrot intake from pre- to post-intervention as the covariate. Pearson's correlation was used to determine any relationships between change in intake of carrot and artichoke from preto post-intervention. To examine the effect of condition assignment on post-intervention intake, ANOVA was carried out with exposure (exposure 10/post-intervention) as within-subject factor and condition as the between-subject factor. Sphericity was not assumed in the analysis and the Greenhaus-Geisser correction was applied. All children tested were included in the analysis. ANOVA was also used to examine the sensory characteristics of the artichoke and carrot puree. SPSS (version 17; SPSS, Inc.) was used for statistical analysis and the $\alpha$-value chosen was $0 \cdot 05$. Omega-sq was calculated to investigate effect sizes for the effect of age and condition on changes in vegetable intake.

\section{Results}

\section{Participants}

Parents provided informed consent for 108 children to take part in the study. Of the 108 recruited, fourteen children were excluded due to food allergies ( $n$ 3) and for being older than 40 months ( $n$ 11). Of the ninety-four children, six children refused to take part in the study, fifteen were excluded due to lack of attendance at nursery and one was removed for incomplete exposures. Table 2 provides characteristics of the children who took part in the intervention.

Out of the potential sample, seventy-two completed the study. Of these, $56 \%$ were girls and $44 \%$ were boys. At baseline, the ages (in months) of the children allocated to each group did not differ, i.e. RE 23.5 (SEM 1.4), FFL 23.4 (SEM 1.5) and FNL 23.68 (SEm 1.7) $(P=0.9)$. Table 3 shows the characteristics of the children in each experimental group according to age (younger: 23 months and younger, older: 24-38 months). To ensure a good representation of ethnic background and socio-economic status, we selected nurseries in a variety of different locations in West and South Yorkshire, UK.

\section{Intake data: baseline, pre- and post-intervention intake (g)}

Baseline intake of artichoke or carrot did not vary between conditions $(P=0.6)$; carrot intake in the respective conditions was: RE was 69.2 (SEM 18.2), FNL 54.2 (SEM 15.4) and FFL

Table 3. Characteristics (age, weight-for-height BMI z-scores) of children in each experimental condition

(Mean values with their standard errors; number of children)

\begin{tabular}{|c|c|c|c|c|c|c|}
\hline \multirow[b]{2}{*}{ Characteristics } & \multicolumn{2}{|c|}{$\mathrm{RE}$} & \multicolumn{2}{|c|}{ FFL } & \multicolumn{2}{|c|}{ FNL } \\
\hline & Mean & SEM & Mean & SEM & Mean & SEM \\
\hline $9-23$ months $(n 40)(n)$ & \multicolumn{2}{|c|}{12} & \multicolumn{2}{|c|}{15} & \multicolumn{2}{|c|}{13} \\
\hline Age (months) & $18 \cdot 3$ & $1 \cdot 2$ & 18 & 0.9 & $15 \cdot 9$ & 1.3 \\
\hline Age range (months) & \multicolumn{2}{|c|}{$10-23$} & \multicolumn{2}{|c|}{$11-23$} & \multicolumn{2}{|c|}{$9-23$} \\
\hline \multicolumn{7}{|l|}{$\operatorname{Sex}(n)$} \\
\hline Boys & \multicolumn{2}{|c|}{5} & \multicolumn{2}{|c|}{6} & \multicolumn{2}{|c|}{2} \\
\hline Girls & \multicolumn{2}{|c|}{7} & \multicolumn{2}{|c|}{9} & \multicolumn{2}{|c|}{12} \\
\hline BMI $z$-score & $1 \cdot 1$ & 0.2 & $1 \cdot 2$ & 0.2 & $1 \cdot 2$ & 0.2 \\
\hline 24-38 months ( $n$ 32) (n) & \multicolumn{2}{|c|}{10} & \multicolumn{2}{|c|}{10} & \multicolumn{2}{|c|}{12} \\
\hline Age at first acceptance test (months) & 29.8 & $1 \cdot 1$ & $31 \cdot 6$ & $1 \cdot 2$ & $31 \cdot 6$ & 0.9 \\
\hline Age range (months) & \multicolumn{2}{|c|}{$26-35$} & \multicolumn{2}{|c|}{$26-38$} & \multicolumn{2}{|c|}{$26-35$} \\
\hline \multicolumn{7}{|l|}{$\operatorname{Sex}(n)$} \\
\hline Boys & \multicolumn{2}{|c|}{$\begin{array}{l}5 \\
5\end{array}$} & \multicolumn{2}{|c|}{7} & \multicolumn{2}{|c|}{7} \\
\hline Girls & \multicolumn{2}{|c|}{5} & \multicolumn{2}{|c|}{3} & \multicolumn{2}{|c|}{5} \\
\hline BMI $z$-score & $1 \cdot 1$ & 0.4 & $1 \cdot 0$ & 0.2 & $1 \cdot 1$ & 0.5 \\
\hline
\end{tabular}

RE, repeated exposure; FFL, flavour-flavour learning; FNL, flavour-nutrient learning. 


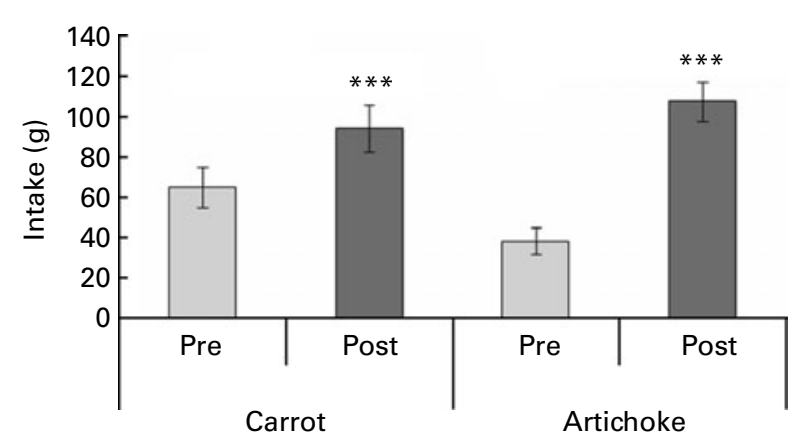

Fig. 1. Absolute intake $(g)$ at baseline (pre-intervention (Pre)) and postintervention (Post, main effect of vegetable). Values are means, with their standard errors represented by vertical bars. ${ }^{* * *}$ Mean value was significantly different from that at baseline $(P<0.001)$.

$72 \cdot 2$ (SEM 15.3) g and artichoke intake was RE $25 \cdot 4$ (SEM 6.3), FNL $37 \cdot 1$ (SEM 10.8) and FFL 53 (SEM 14.5) g.

Overall, baseline carrot intake was significantly higher compared to artichoke $(P=0 \cdot 05)$. Average intake of carrot was $64 \cdot 2$ (SEM 9.8) $\mathrm{g}$ and artichoke was 38.7 (SEM 6.5) g, indicating that carrot was initially favoured over artichoke. A significant main effect of time was observed; intakes of both vegetables increased significantly from pre- to post-intervention ( $P=0.001$ ) (Fig. 1).

Changes in intake. To further investigate this main effect of time, changes in intake $(\Delta)$ were calculated. Artichoke intake increased significantly more than carrot intake (69) (SEM 8.7) v. 29 (SEM 7.2) g $(P=0.001)$. No main effect of condition or vegetable by condition interaction was observed, demonstrating that all methods were equally effective at increasing intake (Fig. 2). Because baseline carrot was higher compared to artichoke, baseline carrot intake was entered in to the model as a covariate. The significant effect of vegetable remained $(P=0.04)$, with artichoke intake increasing more than for carrot intake $(P=0 \cdot 001)$. A significant vegetable by baseline carrot intake interaction was revealed $(P=0 \cdot 01)$ and non-significant effect of baseline carrot intake was found $(P=0 \cdot 7)$. No significant effect of the condition was observed, demonstrating that artichoke increased more than carrot even after controlling for increased carrot intake at baseline.

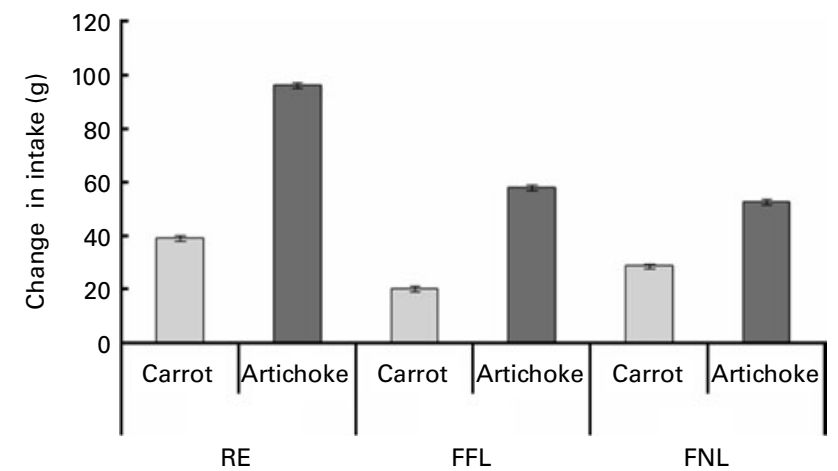

Fig. 2. Change in intake $(\mathrm{g})$ of artichoke and carrot purées (post-intervention minus pre-intervention in each condition). Values are means, with their standard errors represented by vertical bars. ANOVA demonstrated a significant increase in artichoke intake $(P<0.001)$. RE, repeated exposure; FFL, flavour-flavour learning; FNL, flavour-nutrient learning.

\section{Exposure data (intake (g))}

In addition to examining intake from pre- to post-intervention, intake at each exposure was examined in order to investigate the maximum amount of exposures required to increase intake relative to the first exposure. A main effect of exposure number was observed ( $P=0 \cdot 001$ ) (Fig. 3). Pairwise comparisons revealed that intake during exposures 5, 6, 7, 8, 9 and 10 were significantly higher than intake during exposure 1 $(P=0.005)$ (Fig. 3). Intake at exposures 5 through to $10 \mathrm{did}$ not differ significantly $(P=1 \cdot 0)$. No main effect or interactions of condition were found $(P=0.851)$.

\section{Exposure data with changes in carrot intake as a covariate}

Artichoke intake over the ten exposures was investigated, with change in carrot intake from pre- to post-intervention as a covariate. A significant effect of $\Delta$ carrot intake was found $(P=0.001)$ and also a time by $\Delta$ carrot interaction $(P=0.001)$, demonstrating that artichoke intake is related to changes in carrot intake. As expected, a significant effect of time was observed, with artichoke intake increasing over time $(P=0 \cdot 001)$. Controlling for changes in carrot intake, no significant effect of condition remained; RE was 65.3 (sem 7.4), FNL 52.2 (sem 6.9) and FFL 60.9 (sem 6.9) g. A significant positive relationship was found between changes in artichoke and carrot intake $(P=0 \cdot 001)$.

\section{Effect of condition assignment on post-intervention intake}

Intake (g) of artichoke during the last exposure (exposure 10) and post-intervention was investigated to test whether children in the RE group consumed more of the post-intervention version, because this was what they had been offered to consume during the entire experiment. As expected, a main effect of exposure $(P=0 \cdot 001)$ was observed, with post-intervention intake $(102.9$ (SEM 4.1) g) being higher than intake at exposure 10 (64.6 (sEm 8.6) g). No main effects or interactions of condition were found, indicating that condition had no influence on how much was consumed at post-test relative to the last exposure.

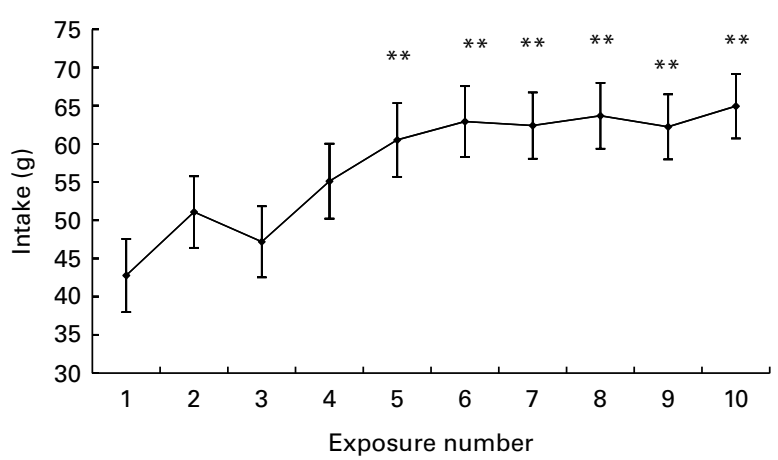

Fig. 3. Average intake (g) over the ten exposures. Values are means, with their standard errors represented by vertical bars. ${ }^{* \star}$ Mean value was significantly different from that at exposure number $1(P<0.01)$. One-way ANOVA demonstrated a significant increase in the intake of artichoke from exposures 5-10 compared to exposure $1(P<0.01)$. 


\section{Follow-up data (n 45)}

Of the seventy-two children taking part in the study, forty-five completed the follow-up (RE $n$ 16, FFL $n 15$ and FNL, $n$ 14). Considering artichoke intake only $(\mathrm{g})$ from pre- to post-intervention measures, artichoke intake increased significantly $(P=0.001)$ from pre-intervention compared to post-intervention and after 3, 4 and 5 weeks post-intervention. Intakes across the entire post-intervention period did not vary significantly. A significant time by condition interaction was observed $(P=0.02)$, demonstrating elevated intake in the RE condition at post-test and follow-up (Fig. 4).

Comparing intake of artichoke over time relative to the carrot control, a significant time by vegetable interaction $(P=0.001)$ was observed. As expected, pre-intervention carrot intake was higher compared to artichoke intake (66 (SEM 13.8) v. 41.2 (SEM 8.8) g). At post-intervention and 5 week follow-up, intake of artichoke was higher than carrot intake (post-intervention: 124.5 (SEM11.6) v. 114 (SEM 15.5) g and 5-week follow-up: $136 \cdot 2$ (SEM 15.9) v. 112.9 (SEm 15.2) g).

Change in intake from pre-intervention to 5 weeks followup was calculated. A main effect of vegetable was found $(P=0.001)$, i.e. overall change in artichoke intake was greater than carrot intake (95.04 (SEM 11.29) v. 46.9 (SEM 13.28) g). A significant vegetable by condition interaction was found $(P=0.043)$ (Fig. 5). Artichoke intake varied significantly $(P=0.024)$ between RE and FFL, whilst no differences in intake were found between RE and FNL and FNL and FFL. Changes in carrot intake did not vary between the conditions $(P=0.37)$.

\section{Baseline, pre- and post-intervention intake (g) with age as} a factor (n 72)

Older children are generally expected to consume more food than younger children; however, older children are more likely to be neophobic ${ }^{(37)}$, so age group was entered into the ANOVA as a between-subjects factor to investigate any main effect or interaction with age on intake $(\mathrm{g})$. Baseline intake of artichoke and carrot did not vary according to condition assignment, and no differences were observed between the three groups for either artichoke or carrot $(P=0.7)$.

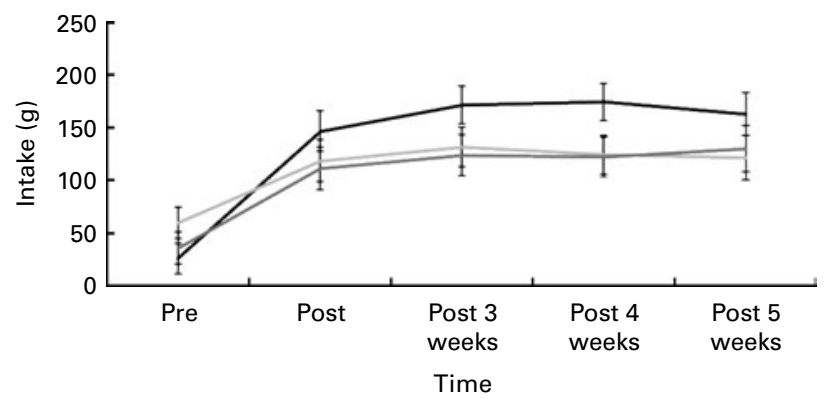

Fig. 4. Intake (g) of artichoke at pre-, post- and 3, 4 and 5 weeks post-intervention. Values are means, with their standard errors represented by vertical bars. Mixed ANOVA revealed a significant time by condition interaction $(P<0.01)$. _ - Repeated exposure; _ , flavour-flavour learning; _ _ flavour-nutrient learning.
No effect of age on intake was observed for baseline intakes $(P=0 \cdot 1)$ and no age by condition $(P=0 \cdot 9)$ or age by condition by vegetable interactions were observed $(P=0 \cdot 2)$.

When pre- and post-intervention intakes were considered, younger children consumed significantly more vegetables than older children $(P=0.004)(95.5$ (SEM 10.1) v. 51.4 (SEM $11 \cdot 1) \mathrm{g}$ ). A significant age by time interaction was observed $(P<0.01)$, with younger children consuming more overall at pre- $(61.7$ (SEM 6.9) v. 39.1 (SEM 10.7) g) and post-intervention (129.2 (SEM 12.21) v. $65 \cdot 6$ (SEM 13.6) g) $(P=0 \cdot 002)$.

Change in intake. When the magnitude of change was examined, as expected, artichoke increased significantly more than carrot $(66.5$ (SEM 8.4) v. 27.4 (SEm 7.2) g) $(P=0 \cdot 001)$ and younger children increased their intake overall compared to older children (67.5 (SEM 8.6) v. 26.4 (SEM 9.6) g). No interactions with age or condition were found.

\section{Non-eaters and plate clearers}

All children were included in the final analysis, as removal of the non-eaters and plate clearers did not make an impact on the results. A total of thirty-four children were classified as regular eaters. Of these, sixteen children were classified as non-eaters and $73 \%$ of these children were from the older group. In all, twenty-two children were identified as plate cleaners and these children were equally split between the young and the older groups. All categories of children were distributed equally across conditions.

\section{Sensory profile}

Concerning sweetness intensity, all samples differed from each other $(P=0 \cdot 0001)$ : as expected, the FFL purée was perceived as sweeter (3.2 (SEM 0.2)) than the other artichoke purées (RE purée, $1.6(\operatorname{sem} 0 \cdot 2)$; FNL purée $1.3(\operatorname{SEM} 0 \cdot 2)$ ). Mean rating of sweetness for the carrot purée was $1 \cdot 9$ (SEM 0·1). For the intensity of 'fattiness', the samples were clearly differentiated $(P=0 \cdot 0001)$ : as expected, the FNL purée was perceived as fattier $(2 \cdot 3(\operatorname{Sem} 0 \cdot 3))$ than the RE $(1 \cdot 7(\operatorname{SEm} 0 \cdot 2))$ and FFL $(1 \cdot 6(\operatorname{SEm} 0 \cdot 2))$

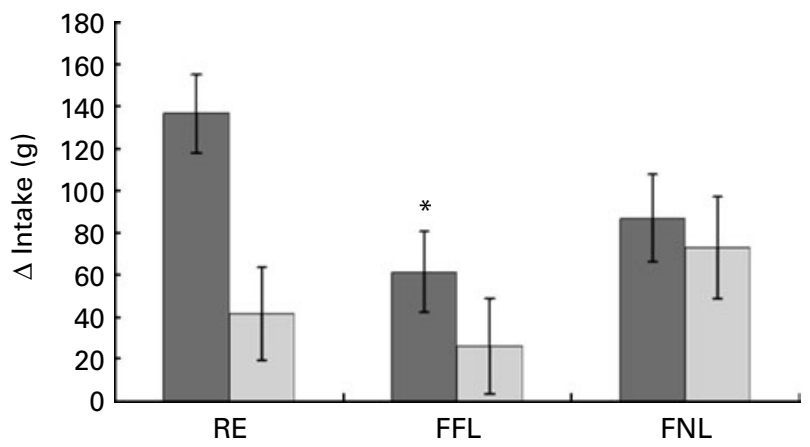

Fig. 5. Changes in intake (g) from pre-intervention to after 5 weeks follow-up ( $n$ 45). Values are means, with their standard errors represented by vertical bars. * Mean value was significantly different from that for RE (artichoke) $(P<0.05)$. Mixed ANOVA revealed a significant difference in change in intake between repeated exposure (RE) and flavour-flavour learning (FFL) $(P<0.04)$. FNL, flavour-nutrient learning. $\square$, Artichoke; $\square$, carrot. 
purées, which were non-significantly different from one another. The carrot purée $(1 \cdot 0(\operatorname{SEM} 0 \cdot 1)$ ) was perceived as being less fatty than the three artichoke purées.

With regard to the other taste attributes, saltiness intensity also varied among samples $(P=0 \cdot 0001)$. The carrot purée was perceived as less salty $(0.7$ ( SEM $0 \cdot 1))$ than the artichoke purée. The FFL purée (1.5 (SEM 0.2)) was perceived as less salty than the RE purée (2.1 (SEM 0.3)) and the FNL purée $(2 \cdot 1$ (SEM 0.2)), which were not different from one another. Concerning sourness and bitterness intensity $(P=0.0001$ and $0 \cdot 01$, respectively), the RE purée was perceived to be more Sour (2.7 (SEM 0.2)) and more bitter (1.1 (SEM 0.2)) than the other purées, which did not differ from each other (for sourness and bitterness, respectively; FFL purée, 1.9 ( $\operatorname{sem~0.2)~}$ and 0.8 (SEM 0.1); FNL purée, 1.9 (SEM 0.2) and $0 \cdot 7$ (SEM 0.1); and carrot purée, 1.5 (SEM $0 \cdot 1$ ) and 0.6 (SEM 0.1)). Samples also differed slightly in terms of umami intensity $(P=0.05)$ : the carrot purée (0.6 (SEM0.1)) was perceived less umami than the artichoke purées, which did not differ from each other (1.1 (SEM 0.1); 1.0 (SEM 0.1); 0.9 (SEM 0.1) for the RE, FNL and FFL purées, respectively).

\section{Discussion}

Results of the present investigation demonstrate that compared to FFL and FNL, RE is sufficient to increase intake of a novel vegetable in pre-school children. In the present study, we demonstrated that after ten exposures to a novel vegetable (artichoke), intake increased from pre- to post-intervention to a greater extent than the change in intake of an already familiar and liked control vegetable (carrot). This effect persists even after controlling for the higher intake of carrot at baseline. Carrot intake was higher than artichoke intake at baseline, suggesting that the children showed increased preference for carrot. However, this did not represent a ceiling effect, as carrot intake post-intervention also significantly increased compared to pre-intervention. Although there was a tendency for RE to result in higher intakes of artichoke in the post-intervention period and up to 5 weeks post-exposure, no significant differences were found among RE, FFL and FNL. These findings demonstrate that all methods were equally as effective at promoting novel vegetable intake. Children consumed similar amounts of each version of the artichoke over the exposure period, demonstrating equal acceptance of the artichoke in each condition. Increases in artichoke intake over the experimental period remained significant after controlling for changes in carrot intake. In addition, the increase in artichoke intake over the experimental period was significantly related to changes in carrot intake from pre- to postintervention. This suggests that response to RE to artichoke reflects a generally positive behavioural tendency towards vegetable intake.

Interestingly, the results show that maximum overall intake was observed from exposure 5, suggesting that five to six exposures might be sufficient to promote intake of a novel vegetable under these circumstances ${ }^{(8)}$. Overall, intake of artichoke remained the same from exposure 5 through to exposure 10, thus no evidence of fatigue was observed.
In confirmation of previous experiments, $\mathrm{RE}$ is effective at promoting increased intake of a novel food ${ }^{(8,13,28-32,38)}$. The aim of the present study was to compare three distinct learning mechanisms, yet, in essence, all children were repeatedly exposed to a target vegetable regardless of its manipulation. Kalat \& Rozin ${ }^{(39)}$ proposed that RE is an effective technique at promoting novel food intake as it allowed for learned safety to be acquired. Repeated positive experience of a novel food encourages associations to develop between sensory characteristics of the food and positive post-ingestive consequences. Techniques such as providing children with a familiar and liked dip might facilitate initial tastings during $\mathrm{RE}^{(8)}$, a technique that might be useful to employ in the initial stages of future experiments. Despite the apparent success of RE, Maier et al. ${ }^{(40)}$ indicated that caregivers tend not to persist in offering a novel food that is perceived to be disliked. In the present context, approximately five offerings significantly increased intake. Whether or not persistent effects are observed with five to six offerings remains to be fully investigated. Recommending five exposures to parents and caregivers might be a more manageable method to encourage intake than suggesting ten repetitions.

Previous research has shown that children have increased preference for foods that are energy dense ${ }^{(13-15)}$. In the present study, weight of artichoke consumed in the FNL condition was non-significantly different to RE or FFL. As would be expected, energy intake did differ (data not shown); the children in the FNL condition consumed more energy on average over the exposure periods compared to the RE and FFL groups, but this did not promote increased intake of the target vegetable post-intervention. The present results support the findings of Zeinstra et $a l^{(16)}$, in that FNL demonstrated no significant advantage.

Children have an innate liking for sweet taste $e^{(41)}$, and so this was exploited for the FNL condition, by sweetening the flavour of the artichoke. Havermans \& Jansen ${ }^{(25)}$ reported increased preference for a target vegetable that had previously been paired with dextrose compared to an unsweetened vegetable in a group of pre-school children. In the present context, there was no advantage of additional sweetness in promoting intake of the target vegetable. Discrepancies in the findings might relate to the study design. Havermans \& Jansen did not measure intake, only rated preference of the vegetables. Also, the sample size in the Haverman's study was smaller, the children were older and the children had six exposures over a more compressed $2 \mathrm{~d}$ period.

In the present investigation, the children were split into two groups, i.e. children aged 23 months and younger and those aged 24 months or older. This criterion was chosen because food neophobia/pickiness is expressed at about 24 months $^{(37,42)}$. The results show that the younger children consumed significantly greater amounts of both vegetables compared to the older children. Several explanations can account for these differences. The most apparent is that the older children were displaying increased neophobic tendencies (non-eaters) and were more reluctant to consume the food offered to them ${ }^{(43)}$. Alternatively, it might be due to the texture of the foods offered. Children in the older 
groups are less familiar with consuming puréed foods than the children in the younger group. Older children have more recent experience of adult 'table food' that differs in texture and appearance to the purées offered, thus amplifying their neophobic response.

The children classified as non-eaters and plate clearers were not responsive to the manipulation, as they consumed either very little or all of what was offered. These children were not from any one condition; thus these characteristics are related to individual differences between the children and not the artichoke. These individual differences might be related to the environment in which they live or due to individual traits that make an impact on eating behaviour. These include exposure to vegetables in the home ${ }^{(44)}$, parental feeding practices ${ }^{(4)}$, temperamental characteristics ${ }^{(45)}$, appetite ${ }^{(46,47)}$ and genetics ${ }^{(48)}$. Future investigations should examine the contribution of these factors to identify which methods are most effective at promoting vegetable intakes in pre-school children.

Limitations of the present study include the use of a relatively small sample size per condition, which might have weakened the strength of the effects observed. However, the present investigation had sufficient numbers to detect large effect sizes for the age on change in artichoke intake $\left(\begin{array}{l}\Omega \\ 0.12)\end{array}\right)$ and small effect sizes, as expected, for change in carrot intake $(\Omega 0.03)^{(49)}$ (data not shown).

It is important to consider that the purée offered might vary in taste compared to when artichoke is offered in its real form (unprocessed). The process of puréeing and adding ingredients such as water or oil might have diluted the taste of the artichoke, thus increasing its acceptability to the children. Future work should extend these findings by offering the vegetables in their pure form. This would ensure that both the taste and texture of the vegetables are experienced and not just the taste in the form a purée. Intake increased to a similar extent in all conditions, and this might have been due to context effects; the presence of the experimenters might have encouraged all children to consume more than usual, thus in future studies parents and caregivers should be responsible for offering the vegetables. In line with this, not all the children included in the study were able to feed themselves, and it is difficult to determine precisely when young children who cannot speak and cannot feed themselves feel satiated. For future investigations, it is suggested that a system for recognising disengagement and satiety cues is used for infants. Finally, a control group was not used in the present intervention; therefore it is difficult to identify whether increases in intake would have been observed in the absence of RE. Nevertheless, when comparing differences in intake over time with RE, the magnitude of effect was greater for the target vegetable (a relatively novel vegetable to most of the children) compared to any change to the control vegetable (a relatively familiar vegetable to most of the children).

In conclusion, there are a number of investigations demonstrating the effectiveness of $\mathrm{RE}^{(8,13,28-33,38)}$ and the effectiveness of this approach at promoting vegetable intake in children. The present study confirms and extends these findings by highlighting the effectiveness of RE as a strategy for promoting the intake of a novel vegetable by comparing three learning mechanisms. RE, FNL and FFL were equally effective at promoting the intake of a novel vegetable in pre-school children. Overall, the results suggest that RE (to any variant) is sufficient to increase intake for up to 5 weeks after the exposure period. In light of the present childhood obesity epidemic, RE is the technique most likely to be endorsed as this method does not require the addition of significant amounts of energy in addition to a regular preparation method, flavouring or indeed reward. More importantly, RE is a simple effective technique that can be used in both the home and in day care settings to improve acceptance of novel vegetables.

\section{Acknowledgements}

S. J. C., S. M. A., E. R., S. N. and M. M. H. contributed to the design of the study. S. J. C. and S. M. A. collected data and contributed to the analyses. M. M. H. and P. B. contributed to the interpretation of the results. E. R. and S. N. were responsible for the product development and production. All authors read and approved the final manuscript. None of the authors declares a conflict of interest. This research has received funding from the European Community's Seventh Framework Programme (FP7/2007-3) under grant agreement no. 245012HabEat coordinated by Dr Sylvie Issanchou. (INRA, UMR 1324, Centre de Sciences du Goût et de l'Alimentation, F-21000 Dijon France).

\section{References}

1. Ransley JK, Greenwood DC, Cade JE, et al. (2007) Does the school fruit and vegetable scheme improve children's diet? A non-randomised controlled trial. J Epidemiol Commun Health 61, 699-703.

2. Anderson A, Porteous L, Foster EH, et al. (2005) The impact of a school-based nutrition education intervention on dietary intake and cognitive and attitudinal variables relating to fruits and vegetables. Public Health Nutr 8, 650-656.

3. Horne PJ, Greenhalgh J, Erjavec M, et al. (2011) Increasing pre-school children's consumption of fruit and vegetables. A modelling and rewards intervention. Appetite 56, 375-385.

4. Gregory JE, Paxton SJ \& Brozovic AM (2011) Maternal feeding practices predict fruit and vegetable consumption in young children. Results of a 12-month longitudinal study. Appetite 57, 167-172.

5. Savage JS, Fisher JO \& Birch LL (2007) Parental influence on eating behavior: conception to adolescence. J Law Med Ethics 35, 22-34.

6. Spill MK, Birch LL, Roe LS, et al. (2010) Eating vegetables first: the use of portion size to increase vegetable intake in preschool children. Am J Clin Nutr 91, 1237-1243.

7. Kral TVE, Kabay AC, Roe LS, et al. (2009) Effects of doubling the portion size of fruit and vegetable side dishes on children's intake at a meal. Obesity 18, 521-527.

8. Anzman-Frasca S, Savage JS, Marini ME, et al. (2012) Repeated exposure and associative conditioning promote preschool childrens liking of vegetables. Appetite 58, 543-553.

9. Johnston CA, Palcic JL, Tyler C, et al. (2011) Increasing vegetable intake in Mexican-American youth: a randomized controlled trial. J Am Diet Assoc 111, 716-720.

10. Spill MK, Birch LL, Roe LS, et al. (2011) Hiding vegetables to reduce energy density: an effective strategy to increase 
children's vegetable intake and reduce energy intake. $A m \mathrm{~J}$ Clin Nutr 94, 735-741.

11. Caton SJ, Ahern SM \& Hetherington MM (2011) Vegetables by stealth. An exploratory study investigating the introduction of vegetables in the weaning period. Appetite 57, 816-825.

12. Brunstrom JM (2005) Dietary learning in humans: directions for future research. Physiol Behav 85, 57-65.

13. Birch LL, McPhee L, Steinberg L, et al. (1990) Conditioned flavor preferences in young children. Physiol Behav $\mathbf{4 7}$, 501-505.

14. Johnson SL, McPhee L \& Birch LL (1991) Conditioned preferences: young children prefer flavors associated with high dietary fat. Physiol Behav 50, 1245-1251.

15. Kern DL, McPhee L, Fisher J, et al. (1993) The postingestive consequences of fat condition preferences for flavors associated with high dietary fat. Physiol Behav 54, 71-76.

16. Zeinstra GG, Koelen MA, Kok FJ, et al. (2009) Children's hard-wired aversion to pure vegetable tastes. A 'failed' flavour-nutrient learning study. Appetite 52, 528-530.

17. Ackroff K, Dym C, Yiin Y-M, et al. (2009) Rapid acquisition of conditioned flavor preferences in rats. Physiol Behav 97, 406-413.

18. Myers KP \& Hall WG (2000) Conditioned changes in appetitive and consummatory responses to flavors paired with oral or nutrient reinforcement among adult rats. Physiol Behav 68, 603-610.

19. Myers KP \& Hall WG (1998) Evidence that oral and nutrient reinforcers differentially condition appetitive and consummatory responses to flavors. Physiol Behav 64, 493-500.

20. Yeomans MR, Leitch M, Gould NJ, et al. (2008) Differential hedonic, sensory and behavioral changes associated with flavor-nutrient and flavor-flavor learning. Physiol Behav 93, 798-806.

21. Mobini S, Chambers LC \& Yeomans MR (2007) Effects of hunger state on flavour pleasantness conditioning at home: flavour-nutrient learning vs. flavour-flavour learning. Appetite 48, 20-28.

22. Brunstrom JM \& Fletcher HZ (2008) Flavour-flavour learning occurs automatically and only in hungry participants. Physiol Behav 93, 13-19.

23. Yeomans MR, Gould NJ, Mobini S, et al. (2008) Acquired flavor acceptance and intake facilitated by monosodium glutamate in humans. Physiol Behav 93, 958-966.

24. Brunstrom JM, Downes CR \& Higgs S (2001) Effects of dietary restraint on flavour-flavour learning. Appetite 37, 197-206.

25. Havermans RC \& Jansen A (2007) Increasing children's liking of vegetables through flavour-flavour learning. Appetite $\mathbf{4 8}$, 259-262.

26. Zajonc RB (1968) Attitudinal effects of mere exposure. J Person Soc Psychol (Monograph Supplement) 9, 2 part 2, $1-27$.

27. Thompson RF \& Spencer WA (1966) Habituation - a model phenomenon for study of neuronal substrates of behavior. Psychol Rev 73, 16.

28. Birch LL, Gunder L, Grimm-Thomas K, et al. (1998) Infants' consumption of a new food enhance acceptance of similar foods. Appetite 30, 283-295.

29. Gerrish CJ \& Mennella JA (2001) Flavor variety enhances food acceptance in formula-fed infants. Am J Clin Nutr $\mathbf{7 3}$, 1080-1085.

30. Sullivan SA \& Birch LL (1994) Infant dietary experience and acceptance of solid foods. Pediatrics 93, 271-277.
31. Loewen R \& Pliner P (1999) Effects of prior exposure to palatable and unpalatable novel foods on children's willingness to taste other novel foods. Appetite 32, 351-366.

32. Pliner P \& Stallberg-White C (2000) 'Pass the ketchup, please': familiar flavors increase children's willingness to taste novel foods. Appetite 34, 95-103.

33. Wardle J, Cooke LJ, Gibson EL, et al. (2003) Increasing children's acceptance of vegetables; a randomized trial of parent-led exposure. Appetite 40, 155-162.

34. Remington A, Anez E, Croker $\mathrm{H}$, et al. (2012) Increasing food acceptance in the home setting: a randomized controlled trial of parent-administered taste exposure with incentives. Am J Clin Nutr 95, 72-77.

35. Baeyens F, Vansteenwegen DEB, De Houwer JAN, et al. (1996) Observational conditioning of food valence in humans. Appetite 27, 235-250.

36. Ahern SM, Caton SJ, Bouhlal S, et al. (2012) Vegetable intake and liking in pre-school children: a cross cultural comparison of three European countries, British Feeding and Drinking Group Annual Meeting Abstracts. Appetite 59, 619.

37. Dovey TM, Staples PA, Gibson EL, et al. (2008) Food neophobia and 'picky/fussy' eating in children: a review. Appetite 50, 181-193.

38. Wardle J, Herrera ML, Cooke L, et al. (2003) Modifying children's food preferences: the effects of exposure and reward on acceptance of an unfamiliar vegetable. Eur J Clin Nutr 57, 341-348

39. Kalat JW \& Rozin P (1973) 'Learned safety' as a mecanism in long-delay taste-aversion learning in rats. J Compar Physiol Psychol 83, 198-207.

40. Maier A, Chabanet C, Schaal B, et al. (2007) Food-related sensory experience from birth through weaning: contrasted patterns in two nearby European regions. Appetite 49, 429-440.

41. Steiner (1979) Human facial expressions in response to taste and smell stimulation. Adv Child Develop Behav $\mathbf{1 3}$ $257-277$.

42. Nicklaus S, Chabanet C, Boggio V, et al. (2005) Food choices at lunch during the third year of life: increase in energy intake but decrease in variety. Acta Paediatr 94, 1023-1029.

43. Cashdan E (1994) A sensitive period for learning about food. Hum Nat 5, 279-291.

44. Jones LR, Steer CD, Rogers IS, et al. (2010) Influences on child fruit and vegetable intake: sociodemographic, parental and child factors in a longitudinal cohort study. Public Health Nutr 13, 1122-1130.

45. Haycraft E, Farrow C, Meyer C, et al. (2011) Relationships between temperament and eating behaviours in young children. Appetite 56, 689-692.

46. Llewellyn CH, van Jaarsveld CH, Boniface D, et al. (2008) Eating rate is a heritable phenotype related to weight in children. Am J Clin Nutr 88, 1560-1566.

47. Llewellyn CH, van Jaarsveld CHM, Johnson L, et al. (2011) Development and factor structure of the Baby Eating Behaviour Questionnaire in the Gemini birth cohort. Appetite 57, 388-396.

48. Cecil JE, Tavendale R, Watt P, et al. (2008) An obesityassociated FTO gene variant and increased energy intake in children. New Engl J Med 359, 2558-2566.

49. Kirk (1996) Practical significance: a concept whose time has come. Educ Psychol Measur 56, 746-759. 